\title{
A New Revolution is Underway
}

$\mathbf{E}$ very second of our lives, we rely on five basic senses: sight, touch, hearing, smell, and taste. Using these senses we craft, improve, and progress as a human race. With respect to sensing, Lord Kelvin famously made the following observation in the 19th century: "If you can not measure it, you can not improve it." Since then, nearly two centuries of groundbreaking research in mathematics, physics, and chemistry created the scientific revolution that has resulted in the technologies surrounding us today. This revolution made it possible to measure and quantify every aspect of the physical world with a much greater accuracy. Today, we have robots in factories that manufacture faster and more precisely than ever before, devices in buildings that measure and adjust heating and air conditioning, and even cars that can drive mostly on their own. Tomorrow, the Internet of Things (IoT) will take this further by seamlessly expanding our sensing capabilities across the globe with only our imagination as a limit.

The IoT refers to the idea of connecting "everything" to the Internet. This will change the landscape of the Internet as we know it today. No longer crowded with computers and data centers, the Internet will be huddled with sensors and actuatorsthe "things." Smartphones, watches, thermostats, ovens, washing machines, fitness trackers, glucose meters, and cars are already part of our lives in the IoT. The proliferation of more connected devices that can sense, act, and communicate opens the door to many new applications. Imagine self-driving cars that communicate to drive closer together or further apart, shoes that warn you about obstacles, or factories and farms that directly connect to shops in order to produce the exact amount of groceries needed for customers. Minimizing waste, optimizing efficiency, and improving the quality of our lives by reducing the number of low-level decisions we need to take will give us back time to do greater things. The IoT is not a far-off dream; it is

\section{We are in the first stages of a new revolution in which technology is becoming a more intricate part of our lives...}

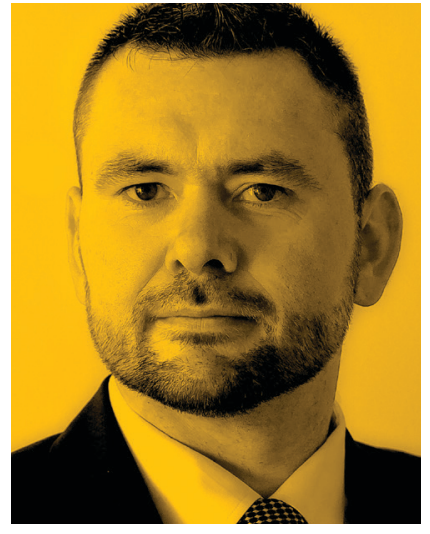

happening all around us. According to ABI Research, by 2020 more than 40 billion devices will be connected to the Internet, with the potential to impact our lives much like the invention of the printing press did in the $15^{\text {th }}$ century.

The current issue of XRDS provides us an overview of IoT, covers groundbreaking research enabling the IoT, and addresses the safety and security issues that need to be solved going forward. We begin with George Hurlburt, who discusses the capabilities and potential for the IoT regarding sensing, thinking, and acting. Hurlburt pleas for a multidisciplinary approach for unlocking the IoT's full potential. Former ACM President and the "Father of the Internet," Vint Cerf, asks what happens if 100,000 refrigerators attack Bank of America. All these new Internet-connected devices could potentially be reprogrammed to send viruses to the network that also hosts the bank's computers. Cerf discusses considerations for the IoT when it comes to interoperability and standardization, as well as security and privacy. He addresses important questions like: What are the tradeoffs between interoperability and long-term profitability of a company, the balance between giving a doctor access to medical information in an emergency while protecting patient data against nefarious uses, and who will, or should, take responsibility when things go wrong?

Things can go wrong not because of maliciousness, but because of miscommunication. With so many new devices we need new ways to interact with them, both for them to understand us and for us to understand them. Jonathan Caras addresses the important topic of interaction with things, and how this can lead to magical experiences. Not only is the way we will interact with the sensors important, but also the capability to personalize the experience. With all the data produced from surrounding devices, we will need to choose and prioritize-very much as we do today with our news feed-what is important to us and what is not. It is the possibility of personalization and interaction everywhere that will create the magic of the IoT.

To have a great user experience we need to enable things 
to communicate efficiently with each other. Low-power devices communicatein delaytolerant networks with constraints very different from ones in the traditional communication networks. The IoT requires extreme power efficiency and an ever-changing network topology, whereas the traditional Internet has many plugged-in machines and routers that connect with physical wires. Alyssa B. Apsel and Enkhbayasgalan Gantsog write about a new communication method that uses little power and finds new devices efficiently. Inspired by the Southeast Asian male firefly, famous for synchronizing its flashes to attract mates, their research involves replicating the way fireflies synchronize flashes to find and communicate with other radios. This method consumes as little as $100 \mu \mathrm{W}$ while transmitting at $150 \mathrm{Kbits} / \mathrm{sec}$ speed, which is four orders of magnitude more efficient than traditional Wi-Fi.

Besides low-power consumption, the devices have to be tiny and cheapwould you pay $\$ 1000$ for a fitness tracker that weighs five pounds? At the same time, they have to be able to compute, communicate, store data, and actuate in the physical world. Creating. such small devices requires tiny electronic components that are big troublemakers. Michael Andersen discusses current trends in IoT development and describes what it takes to construct the physical thing, the enabler of IoT: the ultra-low-power embedded device. Building these ultra-small devices brings new challenges: How do we test and rapidly develop such things? How can we measure and profile their power performance? And, finally, how can we keep prices low?

Imagine trillions of devices sampling every bit of our existence and continuously streaming data. This zettabyte information flow will only be useful if it can be interpreted in a meaningful way. In its essence, the sensor converts the observation of a physical phenomenon into an electrical output with a much greater resolution and speed than a human can. However, we need ways to avail and analyze this information for it to be useful to people. Daniel de Leng takes on this challenge and shares his research about using temporal logic and ontologies to reason about the world in a resource-constrained environment. As a working example, he discusses deploying drones for perimeter monitoring.

Another approach to analyzing all this data is to do it in the cloud. There is often value in aggregating the data of many sensors to find trends and make inferences. A prerequisite for enabling this technology is the privacy of users should be guaranteed. However, a recent study from HP Labs found 80 percent of IoT devices have serious vulnerabilities, 60 percent used unencrypted network traffic, and 70 percent did not require secure passwords. Security is always difficult, especially on resource-constrained devices that are small and easy to lose. Therefore, it becomes a necessity to protect the data rather than the embedded device. Hossein Shafagh discusses the particular challenges that the IoT faces in computation on encrypted data.

The IoT extends far beyond traditional computer science to chemistry, physics, mechanical engineering, and psychology. This poses a challenge: How can we educate the designers and researchers in the multidisciplinary future IoT? Luigi De Russis shares his experience teaching an undergraduate course that provides a holistic IoT development experience. Such courses are a big welcome from industry, which is committing significant resources to IoT research and the development of tools and products.

Finally, we highlight IoT from an industry perspective. Bryant Eastham shares Panasonic's vision of an open and secure IoT. The electronic giant has released 10 years of research and development to the open-source community to accelerate the emergence of the IoT. While, Florian Michahelles and Simon Mayer describe Siemens' efforts to utilize the IoT in industrial automation, energy generation and transmission, control technology, mobility, and medical technology.

The things are here to stay; they will become smarter, more accurate, better connected, and more power-efficient as time passes. Things integrated with reasoning and artificial intelligence have the potential to become our personal shopper and chef; to revolutionize health care (e.g., sensors detecting early symptoms of diseases); to enable smart cars leading to fewer accidents, less congestion, and mitigate pollution; and for countless other applications. We are in the first stages of a new revolution in which technology is becoming a more intricate part of our lives, with the potential to have a profound impact. To achieve this goal, we need to deal with challenges in engineering, human-computer interaction, reasoning, while safeguarding privacy and security.

Are you on board? -Laurynas Riliskis, Issue Editor

\section{Biography}

Laurynas Riliskis is a postdoc at The Stanford Information Networks Group doing research in Secure loT. 